\section{A (Uliniral $\mathfrak{A}$ erture}

ON

\section{TUBERCULOUS CERVICAL GLANDS."}

\section{R. DAVIES-COLLEY, C.M.G., M.Ch., F.R.C.S.,} SURGEON TO GUY'S HOSPIT.IL.

Tuberculosis of the cervical glands is probably alwars secondary to infection by pyogenic organisms, and would soon cease to be one of the common affections of childhoor if better attention were paid to the treatment of septic adenitis in its early stages. Much has been done in recent years to eliminate oral sepsis by early treatment of affections of the tonsils and teeth, and there can be no question that as a result the number of children now seen with tuberculous glands is very much smaller than it was twenty years ago, but the fact remains that it is still very much greater than it ought to be.

It used to be taught that tubercle bacilli reached the cervical glands by direct passage through the lymphatics from the mucous membrane of the mouth and throat, but it is much more likely that they are carried by the blood stream in almost every case. If a direct spread from the epithelial surfaces were a common mode of infection, associated tuberculous lesions of the tonsils, etc., would be frequent instead of comparatively rare. It is fairly safe to say that in a crowded community like London every child has at one period or another tubercle bacilli circulating in its blood stream, which only need the chance coincidence of an area of tissue suitably damaged, either by trauma or sepsis, to enable them to settle down and develop a tuberculoma. Just such an opportunity is provided by the attacks of cervical adenitis so frequent in children, and thus it is hardly an exaggeration to say that every inflamed gland in a child's neck is a potential focus of tuberculous disease.

\section{Anatomy of the Cervical Lymphatics.}

It is, therefore, of the greatest importance to have an accurate knowledge of the paths of lymphatic drainage about the head and neck in order that simple infections of the glands may be traced to their source at an early stage and arrested by suitable treatment before they become dangerous, for it is in the chronic infections that the danger of tubercle arises.

The cervical glands are arranged in two main setsa superficial and a deep-separated from one another by the deep fascia. The superficial set consists of three distinct groups-a suprahyoid group beneath the symphysis of the lower jaw, a submaxillary group beneath the body of the mandible, and a somewhat scattered collection along the course of the external jugular vein situated for the most part between the edges of the sterno-mastoid and the trapezius muscles. Of these groups the first two are concerned in the drainage of the buccal cavity, the teeth, tongue, lips, and face, while the third receives the lymphatics from the scalp, external ear, and the skin of the neck. The deep set of glands is situated along the course of the internal jugular vein in the anterior triangle of the neck, and drains the tonsils, naso-pharynx, pharynx, larynx, trachea, and oesophagus. It also receives communications from the superficial group, and probably a few vessels pass directly to it from the sources normally drained by the superficial glands.

If this very definite grouping of the glands be remembered it should not be difficult to locate in every case the cause of a septic enlargement. In my experience among hospital out-patients the commonest sources of infection in order of frequency are (1) the tonsils, (2) the scalp and external ear, and (3) the teeth. The one most frequently overlooked is the scalp, perhaps because it is not sufficiently recognized that the presence of pediculi is quite enough

* This lecture was delivered some two years ago, and I publish it now because there seens to be a growing tendency to regard tuberculou calling for treatment by operation; a view which I consider to be wron and likely to become dangerous if widely taught and acted upon. to cause enlargement of the glands without any obvious cutaneous lesion.

It is unnecessary to go into details of the treatment of these infections, which is simple enough as a rule, and I would only emphasize again the importance of dealing with them properly and with energy. It is, of course, possible to be too energetic, and the wholesale sacrifice of tonsils which is practised by many at the present time is, I think, an instance of quite unnecessary and probably harmful thoroughness. Hyperplasia of the lymphoid tissue in the mouth is a normal process in childhood, and undoubtedly serves a purpose, though it may not yet be understood exactly what this purpose is; and the still recent discoveries of the value of the secretions of the various ductless glands should warn us against labelling as superfluous any organ the precise function of which is not known. Doctors, and, I am afraid, particularly the medical officers of schools, are apt to overlook this fact, and it cannot be too strongly urged that to condemn tonsils on the strength of one $\mathrm{cr}$ two attacks of acute tonsillitis with temporary swelling of the glands is entirely unjustifiable and reprehensible. As far as the glands are concerned the tonsils should only be removed if the glandular enlargement persists between the attacks of tonsillitis and after less radical measu:es of treatment have been given a thorough trial and have proved unavailing.

\section{Diagnosis.}

The diagnosis of tuberculous glands is fortunately, as a rule, a simple matter. Tuberculosis is the only common cause of persistent enlargement of the cervical glands in the absence of a definite focus of infection. Simple adenitis quickly subsides after removal of the source of infection, and $I$ have not found it in any child on whom I have operated with a previous diagnosis of tuberculous glands. Glandular enlargement from Hodgkin's disease and other general lymphatic diseases is generally easily distinguished by the multiplicity of the glands affected, and in any case, if there be a doubt, the question can be settled without difficulty by removing a superficial gland for histological examination.

Clinically the cases fall into three groups or stages of infection, and from the point of view of treatment it is convenient to consider these three groups separately.

1. In the first or early stage the glands are discrete and can easily be counted; the muscles and skin are not infiltrated, though even from the first the glands may be adherent to one another and their mobility impaired. The earliest to be affected are almost always the glands in the upper part of the neck, but as time goes on the infection tends to spread downwards until a chain of nodules may be felt extending from the base of the skull to the clavicle. As a rule they are firm in consistence, though if they have been for long as large as a filbert nut there will almost certainly be central caseation in some of them and softening may be apparent, but if so the pus is confined within the capsule of the glands and has rot spread to the connective tissue around them. Sometimes they remain for a long while in a state of latency, but there is generally a tendency to periodic outbursts of activity in which they swell up and become painful. Each succeeding exacerbation leaves them a little larger than they were before, and there is always a danger that during one of these attacks a cervical abscess may form.

2 . In the second stage, the stage of abscess, the suppuration has involved the neighbouring connective tissue, especially the sterno-mastoid muscle and the subcutaneous tissue over it, in which collections of pus have formed. The glands near the abscess are no longer palpable as distinct entities, but are matted together in an indefinite mass which is fixed to the structuies round it and is often extremely terider.

3. In the third stage, which follows quickly upon the secend, the abscess has burst through the skin, leaving one or more discharging sinuses. The persistence or not of this stage depends upon whether the gland or glands from which the abscess started have been able completely to discharge their contents into it. If not, persistent sinuses will follow with the gradual development of contracting and disfiguring scars, and in some cases a spreading tuberculous infection of the skin.

Treatment of First Stage.

There are, practically speaking, two methods of dealing with tuberculous glands-operative and non-operative. The first aims at complete removal of the infected tissue 
by excision, while the object of the second is so to raise the patient's resistance that he is able to deal with the infection himself-in other words, to promote a natural cure. I have said that there is a tendency at the present time to teach that the non-operative method gives better results than the operative. I am entirely in disagreement with this view, and my main purpose in this lecture is to endeavour to establish the case for early excision. There are four main considerations by which we may compare the two methods:

1. The completeness of the cure achieved.

2. The length of time needed for the treatment.

3. The dangers resulting from it.

4. The accessibility of the treatment to individual cases.

1. A complete cure is claimed by the advocates of both methods, but $I$ must confess that $\mathbf{I}$ find it hard to believe that tuberculous foci are destroyed by expectant treatment as completely as is generally taught. I so frequently see patients who have had "glands" in childhood which have lit up again in after life that $I$ think it is much more probable that though the obvious enlargement of the glands may subside the tubercles are merely reduced to a state of quiescence, and that for a very long while after this apparent cure the bacilli remain alive and ready to recrudesce if given a favourable opportunity. After all, this is only what we see constantly happening with tuberculous foci in other situations, and it is unlikely that in the cervical glands the life of tubercle bacilli is shorter than elsewhere. The advocates of expectant treatment, it seems to me, draw too sweeping conclusions from the effects of non-operative treatment of tuberculosis of the spine and joints and the bad results which operative surgery has given in both these situations in the past. But here, surely, the conditions are not in the least comparable, for in spinal tubercle excision of the diseased tissue is, from the nature of things, impossible, and surgery is limited to the evacuathon of pus and to the immobilization of the spinal joints, while in the case of tuberculous joints any attempt at excision is of necessity bound to upset their delicate mechanism and to result in a fixed or an unstable limb; and $I$ have no doubt that if a radical operation could be devised which held out a hope of a good functional result the tedious process of waiting for a natural cure would very soon become a thing of the past. With early tuberculous glands in the neck the case is entirely different. A complete excision of all infected tissue can be made without interference with function and brings with it a sense of security in the knowledge that no tubercles are left to infect other glands in the neighbourhood.

2. The length of the treatment is perhaps not of such vital importance as the completeness of the cure, and no doubt for this reason little point is usually made of it. It is, however, of considerable importance, because, apart from the tedium and necessary expense of a long course of treatment, it must be remembered that the age at which tuberculous glands are most prevalent is also the age at which education should be taking a prominent part in a child's life, and any interference with the normal order of things is bound to hamper educational facilities; besides, removal of a child for a long period from its family is not as a rule desirable either for the child or for its parents. In older subjects the interference with their work makes time of still greater importance. There can be no question as to which of the two methods of treatment requires the longer time for its completion. Expectant treatment, if it is to have any chance of success, must be continued for many months or even years, while after excision a few weeks' convalescence by the sea are usually enough to restore complete health.

3 . The dangers of expectant treatment are those which must inevitably arise from the constant presence of a tuberculous focus in any part of the body-that is, local spread of infection and suppuration, metastatic involvement of distant organs, and general tuberculosis. It is true that as long as the tuberculous process is in a state of quiescenceand the object of expectant treatment is to keep it so-the danger of complications is small, but with glandular tubercle it is not easy to ensure quiescence, however careful the treatment. $\dot{A}$ cold or a sore throat is quite sufficient to rouse the bacilli to a burst of activity, and it is quite impossible to avoid trivial ailments of this sort, no matter what precautions are taken. It stands to reason, therefore, that the longer the primary focus remains the greater is the risk of one of these complications developing. Fortunately, both metastasis and general dissemination are rare with tuberculous glands, but that is no excuse for ignoring them. Local spread of infection and suppuration may not in themselves be so dangerous, but there can be no question that they immeasurably increase the difficulties of treatment and sometimes make a complete cure impossible. Gland to gland extension is not altogether without serious consequences, for when the lower cervical glands are reached it is but a short step from them to those in the mediastinum, and I know of at least two instances of direct involvement of the apex of the lung by tuberculous glands in the posterior triangle. The unpleasant results of suppuration are known to all and need no detailed description.

Apart from the natural antipathy which the general public has to any form of cutting operation, a fecling which is easily understood but is for the most part purely sentimental and unreasoning, there are three obvious disadvantages to operative treatment: the risk of the anaesthetic, the resulting scar, and the possibility of injury to important nerves.

The danger from the anaesthetic is so small nowadays that I think it may be disregarded, whilst in an early case the scar left by the operation, if the incision has been carefully placed, is usually almost invisible after a very short time, and in the hands of a competent operator injury to important nerves should be out of the question. So I think it may justly be claimed that the risks from the operation are trivial when compared with those of waiting for a natural cure.

One other objection I have heard raised to operation is that excision of glands in the neck is useless because in tuberculous cases those in the mediastinum are always likely to be involved as well, and as these cannot be reached by the surgeon no operation can hope to achieve more than a partial removal of tuberculous tissue. I know of no reason to suppose that there is a particle of truth in this statement. All our experience goes to show that in the early stage only the upper cervical glands are usually tuberculous, and that it is by a gradual downward spread that the lower cervical and the mediastinal glands become infected.

4. Medical treatment, if it is to have any chance of success, must be carried out in ideal surroundings and under strict medical supervision-conditions which are by no means easily obtained in this country except by those who are able to pay highly for them. The vast majority of tuberculous gland cases occur among the poor in the large towns, and for these treatment in their homes is useless. Institutions for the treatment of surgical tuberculosis are few in number, and there are so many calls upon their limited accommodation by the more urgent types of tuberculosis that the chances of any but the worst gland cases gaining admission to them are small. The average surgical convalescent home is not suitable for cases of tuberculous glands, because the medical supervision at them is not sufficiently strict, and I can remember many instances of having to open cervical abscesses in children who had been sent to convalescent homes in the hope that their glands would clear up under the influence of fresh air and sunshine. Convalescent homes, too, owing to the demand on their beds, are rarely able to keep gland cases long enough to do siny lasting good. So that while it is not a djfficult matter for the well-to-do to obtain surroundings favourable to medical treatment, for the poor it is only possible in a very small percentage of the total cases. On the other hand, accommodation in hospitals is easily found for children requiring operations, and after excision of tuberculous glands they can safely be sent to any convalescent home without fear of untoward complications developing.

The foregoing considerations apply particularly to those cases in which only a small group of glands is tuberculous, and $I$ think it must be admitted that for them on every count the case for operation is a stronger one than that for expectant treatment, and that opèration should therefore be regarded as the method of choice. Exceptions way, 
perhaps, be made in the case of the well-to-do, with whom the risks of waiting are relatively small, but even with them the length of time taken by expectant treatment is so great that operation is preferable.

When the involvement of glands is widespread and the disease has reached the lower nembers of the cervical chain, the question of treatment is harder to decide, because the golden opportunity for excision has passed, and not only are the operation risks, especially that of injury to the spinal accessory nerve, increased, but also the resulting scar is apt to be large and unsightly. In cases of this sort, unless there is some special reason for hurry, medical treatment should be given an extended trial, in the hope that even if not completely cured the glands may be so much reduced that a subsequent operation for their removal may be a relatively easy and safe procedure. But it must be clearly understood that extensive involvement of glands is a state of things which is easily prevented and only occurs as the result of pursuing the hopeless policy of waiting during the earlier stage, when the disease is localized.

I do not propose to discuss in detail the operations for excision of glands. Suffice it to say that there are two points which are essential : (1) all obviously enlarged glands must be removed; and (2) the incisions must be placed in such a way that they will be least noticeable when healed. Both of these essentials are readily secured if the case be taken early. If it be left until adhesions have formed, the difficulties may be such as to tax all the skill of the most experienced operator.

One is sometimes confronted with a case in which softening tuberculous glands are associated with tonsils which obviously require extirpation. Now, if the tonsils are removed in the hope that the glands will subside there is a very considerable danger that the operation in the mouth may precipitate a state of suppuration in the neck and lead to the formation of a cervical abscess. To avoid this unfortunate event it is best in these cases to remove the glands and the tonsils at the same time. The double operation has no deleterious effect on the healing of the wound in the neck, and the patient is thereby saved, not only the risk of a cervical abscess, but also the trouble and expense of a second operation.

\section{Treatment of the Second Stage.}

My remarks on the dangers of non-operative treatment during the first stage apply with still greater force when suppuration has occurred. It is true that aspiration, or repeated aspiration, of tuberculous pus may cause an abscess eventually to dry up, but the process is so slow and so uncertain in its result that $I$ do not think this method of treatment is justified. Operation, to my mind, is clearly called for, and it only remains to consider how extensive an operation is advisable. It is obviously best, when possible, to excise the whole area of diseased tissue, and if the abscess is small and the mass of glands is not large or closely adherent to the surrounding structures this can usually be done without much risk. But if the abscess is a large one, and particularly if there is, as is often the case, a considerable amount of surrounding cellulitis, the risk of injury to important nerves by an attempted excision is so great that it is best not to attempt it. The safer course is to open the abscess widely, and then, having thoroughly scraped the walls of the cavity, to search for the opening by which it communicates with the caseating gland. This can generally be found and through it the semi-solid caseous material can be removed from within the capsule of the gland by means of a sharp spoon-a most important step, for unless the contents of the gland itself are evacuated a discharging sinus is cortain to result. The wound is then sutured with a small drain at its lower end, which is removed after two days, by which time any oozing from the abscess wall will have ceased. Healing is usually rapid, and if only one or two glands are caseous no further operation may be required. In any case the cellulitis quickly subsides and any remaining tuberculous glands or infiltrated muscle can be excised, if necessary, at a later date.

\section{Treatment of the Third Stage.}

When sinuses have formed and the suppuration has reached a chronic stage, the only satisfactory course is to excise the whole mass of tuberculons tissue, and this should be done unless the area of disease is so wide that its excision would leave a gap too large to close. But it must be remembered that at this stage the tough adhesions between the glands and the neighbouring structures will have made the operation a very difficult one, which should not be undertaken by anyone who has not had a large experience of cervical surgery. If the diseased area is too large for excision it is possible, by opening up the sinuses and scraping out the cascous material from the glands to which they lead, to get them to heal, but to obtain a complete cure this must be followed up by a long course of heliotherapy and, if the skin is involved, $x$-ray treatment.

\section{THE DIAGNOSIS OF MALIGNANT DISEASE OF THE LARGE INTESTINE.*}

\section{BY}

HAMILTON DRUMMOND, F.R.C.S.ED.,

SENIOR ASSISTANT SURGEON, ROYAL VICTORIA INFIRMARY, NEWCASTLEUPON-TYNE.

I FEeL encouraged to draw attention to this subject for several reasons: first, because it is a common condition, and secondly, because recent advance in methods of diagnosis and treatment have greatly improved our chances of dealing radically with cancer of the large bowel. Moreover, I have been much impressed, during the last few years, by the late stage at which these cases come to seek surgical advice. That the surgeon does not see these until a late stage-often too late-is generally admitted, and the fact is due to one of several reasons-namely, that the patient ignores the symptoms caused by the disease; that the symptoms are so slight as not to give rise to any trouble until the disease is advanced; or that the symptoms, whether slight or severe, have not impressed the practitioner sufficiently to lead him to make a thorough examination of his patient.

Mr. Wilkie of Edinburgh recently, in an address to the Newcastle-upon-Tyne and Northern Counties Medical Society on diagnosis in surgery, made the following statement, with which I entirely agree:

"Diagnosis is the crux of modern surgery, and that the latter is now attended with so much success is due, in $\mathrm{my}$ opinion, to the improved diagnosis of the general practitioner much more than to the advance in surgical methods and technique, great as these have the advance in surgical methods and technique, great as these have
been. One has but to consider the results of the operative treatbeen. One has but to consider the results of the operative treat-
ment of urgent conditions such as perforated ulcers of the stomach and duodenum and acute appendicitis to realize this fact. The many patients whose lives are now saved by prompt operation should bestow their gratitude on the men who made the early diagnosis, not on the surgeons whose task was made easy."

This is quite in keeping with what occurs in the Royal Victoria Infirmary at the present day, which forms a striking contrast to the time, a few years ago, when $I$ was house-surgeon, when it was common experience that the majority of cases of appendicitis presented themselves with abscess or general peritonitis, while now the pathology is nearly always limited to the appendix.

The diagnosis of carcinoma of the colon has certainly not kept pace with these cases of abdominal emergency. I have no hesitation in saying that the most successful cases of operation for cancer of the large bowel which have come under my care have been sent by a few enthusiastic practitioners who seldom miss an opportunity of making a complete examination, whenever symptoms, however slight, show themselves.

It is to the necessity of early diagnosis I wish to draw special attention, and to emphasize the fact that without the co-operation of the practitioner this is impossible; and that it is upon him the fate of the patient must largely. depend. It is for him to say in doubtful cases that further investigation by one or other of the special diagnostic methods is essential to success in treatment.

What are the points of greatest importance that assist us in the diagnosis of a carcinoma of the large intestine, before laparotomy is performed? If a student be asked this question he will often give the following answer: A bismuth

* An address delivered before the Hartlepool Division of the British *An address delivered before the Hart
Medical Association on January 31st, 1924. 